\title{
La galaxie des pédagogies alternatives, objet d'étude des sciences humaines et sociales
}

\section{Marie-Charlotte Allam et Sylvain Wagnon}

\section{(2) OpenEdition \\ Journals}

Édition électronique

URL : http://journals.openedition.org/trema/4159

DOI : $10.4000 /$ trema.4159

ISSN : 2107-0997

Éditeur

Faculté d'Éducation de l'université de Montpellier

Édition imprimée

Date de publication : 1 novembre 2018

ISSN : 1167-315X

Référence électronique

Marie-Charlotte Allam et Sylvain Wagnon, «La galaxie des pédagogies alternatives, objet d'étude des sciences humaines et sociales », Tréma [En ligne], 50 | 2018, mis en ligne le 05 novembre 2018, consulté le 23 septembre 2020. URL : http://journals.openedition.org/trema/4159 ; DOI : https:// doi.org/10.4000/trema.4159

Ce document a été généré automatiquement le 23 septembre 2020

Trema 


\title{
La galaxie des pédagogies alternatives, objet d'étude des sciences humaines et sociales
}

\author{
Marie-Charlotte Allam et Sylvain Wagnon
}

1 Depuis plusieurs années, les articles sur les pédagogies " alternatives » se multiplient dans la presse grand public et les magazines de vulgarisation scientifique. Cette thématique semble faire recette et rencontrer un écho grandissant dans la société. Il nous a donc semblé nécessaire, par ce numéro de Tréma, d'entreprendre une analyse scientifique critique d'ensemble de cette galaxie des pédagogies alternatives. La lecture de ces très nombreux articles de presse, et en particulier des derniers numéros de Sciences Humaines et de Philosophie Magazine, illustrent des dynamiques à l'œuvre dans le monde des pédagogies alternatives, et qu'il convient d'interroger avec les outils des sciences sociales ${ }^{1}$.

2 Tout d'abord, les deux revues mettent au jour l'existence d'un conflit de valeurs entre les différents courants se revendiquant comme une "alternative » à l'école traditionnelle. On perçoit un clivage entre les courants « historiques » de l'Education nouvelle liés aux figures pédagogiques de Célestin Freinet, Ovide Decroly, Rudolf Steiner et Maria Montessori et de l'autre de «nouvelles » formes éducatives autour des écoles démocratiques, de l'instruction en famille ou de l'enseignement privé confessionnel $^{2}$. Une lutte politique pour représenter «l'alternative» est perceptible entre ces deux courants. Le succès de la pédagogie Montessori est symptomatique de ce «glissement» idéologique. Pédagogie d'éducation nouvelle, elle s'est imposée aujourd'hui comme «l'esprit » et la référence des nouvelles pédagogies alternatives ${ }^{3}$. Ce clivage mérite que nous nous y intéressions et que nous mettions en avant ce débat politique des finalités des pédagogies alternatives.

Ensuite, la montée de ces nouvelles tendances pédagogiques a redimensionné le débat, parfois artificiel, entre «pédagogues » et « républicains » des années 1980. On assiste aujourd'hui à la cristallisation d'un nouveau clivage entre ceux que Philippe Meirieu désigne comme les «antipédagos», militants d'une école «mythifiée » et 
"hyperpédagos ", artisans d'une idéologie familiariste ${ }^{4}$. Les nouveaux enjeux éducatifs concernent moins la manière d'acquérir un savoir - la contradiction entre «transmission» et «émancipation" - que la posture de l'adulte envers l'enfant. «Autorité » et «bienveillance » constituent deux nouveaux pôles autour desquels se positionnent les nouvelles pédagogies alternatives ${ }^{5}$.

4 Le dernier phénomène, et il est indissociable des deux autres, c'est la prégnance des arguments issus des sciences cognitives dans le discours sur l'éducation ${ }^{6}$. Un renouvellement des modes de légitimation scientifique des pédagogies alternatives s'est opéré. La sociologie et la psychologie naissantes avaient accompagné l'émergence de l'Education nouvelle au tournant du $20^{\mathrm{e}}$ siècle. Désormais, les savoirs scientifiques enrôlés dans ces discours réformateurs ont changé ; l'heure est à la déconstruction des mécanismes du cerveau et aux émotions, aux neurosciences et à la psychologie positive. Avec ces nouveaux savoirs, c'est l'analyse des problèmes éducatifs - de l'échec scolaire, de l'ennui, ou bien de la crise de l'école - qui change. Les relations de causalités, les interprétations, la désignation de responsables de ces problèmes ou la formulation de solutions en sont complètement bouleversées. D'où la réticence de certains sociologues face à la nomination de neuroscientifiques au plus près des décideurs politiques de l'Education nationale.

5 Ces constats ne sont pas anodins et appellent une réflexion sur les acteurs qui soutiennent les pédagogies alternatives, sur les fondements politiques et philosophiques de leurs discours et leurs implicites, et sur la manière dont ils se diffusent dans le corps enseignant. C'est une question épineuse, du fait de son insertion dans les débats politiques actuels, mais d'autant plus cruciale. Les caricatures, les descriptions réductrices et approximatives, les analyses hagiographiques ou outrageusement critiques qui entourent les pédagogies alternatives rendaient nécessaire une clarification des fondements et des finalités politiques des différents mouvements de cette nébuleuse. Construire une analyse scientifique et critique des pédagogies alternatives a donc été l'un des premiers objectifs, et l'une des premières difficultés, des initiateurs de ce numéro. Or, si les pédagogies alternatives sont très présentes dans les médias, elles restent encore aujourd'hui un point aveugle de la recherche en France. La revue Spirale avait publié un dossier pionnier sur ce même thème en 2010 (Casanova \& Carra, 2010). La comparaison entre les deux numéros, à huit ans d'intervalle, illustre la mutation et l'ascension de la galaxie des pédagogies alternatives. Une décennie plus tôt, l'enjeu était de déterminer les points communs d'un ensemble de discours et de pratiques (Casanova \& Carra, 2010b). Les exemples étaient principalement tirés de la pédagogie Freinet et de l'Education nouvelle ${ }^{7}$. Aujourd'hui, l'essor du phénomène Montessori, des écoles inspirées du mouvement Sudbury, ou de l'importance d'un Pierre Rabhi, viennent rebattre les cartes et modifier en profondeur l'analyse des pédagogies alternatives pédagogiques.

6 Dans ce numéro de Tréma, notre premier souci était de montrer que derrière la référence commune à une "alternative " se cache des réalités différentes et parfois antagonistes. Ce constat soulève des choix méthodologiques, théoriques, et politiques. En effet, comment dessiner les contours d'un monde qui regroupe en son sein les courants d'Education nouvelle, les écoles dites "démocratiques", les militants de l'instruction en famille ou ceux de l'enseignement privé, religieux ou traditionnalistes ? Est-il souhaitable et possible de rechercher les principes fédérateurs de courants que tout semble opposer, à l'exception de leurs utilisations du terme d'alternatives? 
L'usage même du terme « d'alternative » n'est-il pas un leurre ? S'agit-il d'une nouvelle forme pédagogique - ce que l'usage du lexique de l'alternative semble vouloir souligner, mais que la marginalité numérique rend peu crédible a priori ? Quelles sont les finalités de ces écoles et courants pédagogiques qui se disent alternatifs? Les acteurs de cette galaxie n'ont-ils pas, eux aussi, besoin d'une clarification éducative et politique de leurs actions. Sont-ils des aiguillons de l'école publique? Sont-ils au contraire les fossoyeurs d'un "creuset commun » et les militants d'un repli sur soi, d'une libéralisation et d'une privatisation de l'éducation? Nous nous devions de dénouer les nœuds historiques, sociologiques et pédagogiques pour faire de la question des pédagogies alternatives un objet d'étude des sciences humaines et sociales.

7 La difficulté est bien d'analyser des invariants, des principes fédérateurs pour des mouvements qui ne forment pas un groupe compact loin de là, mais plutôt une nébuleuse. La métaphore de Christian Topalov est pertinente, dans une certaine mesure pour analyser, cet "univers fini mais au contours indécis", cet "ensemble d'objets organisés en systèmes partiels» (Topalov, 1994, p. 13). En effet, il ne s'agissait non pas d'en délimiter les frontières, par définition insaisissables, mais d'en saisir les dynamiques internes, les divergences et les logiques fédératrices. Il se joue, au cœur de cette nébuleuse, une lutte - pour le moins concurrentielle - entre différents groupes qui ambitionnent d'incarner "l'alternative légitime" et qui portent atteinte au monopole de l'école d'Etat sur l'éducation des enfants. Néanmoins, le terme galaxie nous a semblé plus adéquat car ces courants ne représentent aucunement une mouvance commune mais bien plutôt des ilots et des archipels. La variété de tous ces courants nécessitait tout d'abord une réflexion sur les contours - mouvants - de ces alternatives. C'est tout l'enjeu de la contribution de Sylvain Wagnon. Une cartographie élaborée par Hélène André vient éclairer les articulations, relations entre acteurs, mouvements, groupes de pression et lobbys qui gravitent autour des pédagogies alternatives et qui en sont une des caractéristiques.

Mais il nous fallait également analyser la question de leurs émergences, de leurs mutations, de leurs motivations et de leurs finalités. Nous donc avons pris au sérieux ce terme d' " alternative ", et abordé ces différentes expériences en interrogeant l'horizon éducatif, politique, philosophique et scientifique qu'elles esquissent. Marie-Charlotte Allam propose une réflexion sur la médiatisation de ces "alternatives ». La période 1970-2000 apparait bien primordiale pour comprendre le « tournant » du début du XXIe siècle avec l'apparition de nouvelles expériences éducatives alternatives.

Ce numéro est issu d'un appel à communications. Le nombre d'articles, en proportion, qui portent sur la pédagogie Montessori témoigne de son importance et de son statut de «vitrine» des pédagogies alternatives. Référence incontournable, comment la pédagogie Montessori ou « l'esprit Montessori » est sortie en moins d'une décennie de sa tour d'ivoire pour irriguer l'ensemble de pédagogies alternatives? Qu'on le veuille ou non, chacun semble se positionner pour ou contre cette pédagogie. Il fallait donc faire un point scientifique et les trois contributions de Serge Franc, Berengère Kolly et Chrystel Huard nous aident à ce travail.

Delphine Patry et Sylvain Connac étudient successivement deux notions centrales dans le discours des pédagogies alternatives: l'autonomie et la coopération. L'autonomie apparait, comme le montre Delphine Patry comme le graal de toute éducation mais également un enjeu politique majeur. Sylvain Connac s'intéresse à la coopération outil des pédagogies actives mais aussi critère de compréhension des finalités éducatives. 
11 Il était aussi important de demander à un universitaire à la fois spécialiste des pédagogies actives et militant engagé dans les combats politiques et éducatifs actuels de répondre à nos interrogations. Cet entretien de Philippe Meirieu a eu lieu quelques mois avant la parution de La Riposte, son dernier ouvrage. Percutant, il entre de plein pied dans les débats actuels et montre, s'il était encore nécessaire, l'importance de notre sujet d'étude. Son analyse stimulante offre une mise en perspective de certains enjeux majeurs de cette question vive que représentent les pédagogies alternatives aujourd'hui.

\section{NOTES}

1. Les deux revues consacrent en effet leur dossier de rentrée à l'éducation et à la recherche d'une autre école. "Eduquer, le conflit des modèles », Sciences Humaines, ${ }^{\circ}$ 307, Octobre 2018 ; «L'éducation nous rend-elle meilleure ?", Philosophie Magazine, n 122, septembre 2018.

2. Béatrice Kammerer, "Ecole publique, un monopole contesté ", Sciences Humaines, op. cit., p. 32-39.

3. Martin Duru et Alexandre Lacroix, «Une autre pédagogie est possible », Philosophie Magazine, septembre 2018, p. 46-53.

4. Meirieu Ph (2018). La Riposte. Paris : Autrement.

5. Hugo Albandea, «Autorité et bienveillance, des valeurs en tension », Sciences Humaines, $\mathrm{n}^{\circ}$ 307, octobre 2018, p. 32-35.

6. Michel Eltchaninoff, « Notre dernière utopie », Philosophie Magazine, op. cit., p. 42-45; Victorine de Oliveira, «Le cerveau au tableau », Philosophie Magazine, op.cit., p. 55-57.

7. On compte en effet quatre articles sur la pédagogie Freinet et un de Laurent Gutierrez sur la Ligue Internationale de l'Education nouvelle.

\section{AUTEURS}

\section{MARIE-CHARLOTTE ALLAM}

Laboratoire Pacte, Université de Grenoble

SYLVAIN WAGNON

Lirdef, Faculté d'Education, Université de Montpellier 